\title{
The EUSO program to study UHECR from space: status and perspectives.
}

\author{
M. Casolino* \\ INFN and RIKEN \\ E-mail: casolino@roma2.infn.it
}

The study of Ultra High Energy Cosmic Rays (UHECRs) offers unique possibilities to probe the energies currently inaccessible by man-made accelerators. The last years have shed light on several characteristics of these particles, but - due to their extremely low flux - their origin, nature and acceleration mechanisms are still unclear.

Space-based observations have a potential for a large increase in statistics and capability to cover the whole sky, allowing for a direct comparison of spectra and direction of arrival from different regions and sources. Even a relatively small detector, with the exposure of a few times that of Auger, would be able to clarify the differences between the Northern and Southern sky, confirm the existence of TA hot spot, and measure multipolar anisotropies with high precision. To achieve this goal within the constraints of power, mass, size and bandwidth of space detectors, a number of novel technologies - from optics to sensors, front-end and read-out electronics - have been developed over the years. Several scaled versions of a large area space-borne telescope have been built: in August 2014 a balloon flight (EUSO-BALLOON) was successfully launched from Timmins (CA); since February 2015 a ground based detector (EUSO-TA) has been operating at the Telescope Array site in Utah. In 2017 a Super Pressure Balloon (EUSO-SPB) flew from Wanaka, New Zealand. In parallel, a series of missions have been realized by the Moscow State University to study the UV background and transient phenomena from night-time Earth: the Tatiana-1 and -2, Vernon, and TUS satellite-borne detectors. The next steps of the JEM-EUSO program include: a) MINI-EUSO - a detector to be placed inside the International Space Station (2017); b) A second SPB flght, c) K-EUSO - the first reflector based mission to perform UHECR science from space, paving the way to d) POEMMA, a Multi-Messenger devoted mission. In this work we discuss the current status of research and plans of the JEM-EUSO program.

35th International Cosmic Ray Conference - ICRC2017

10-20 July, 2017

Bexco, Busan, Korea

${ }^{*}$ Speaker. 


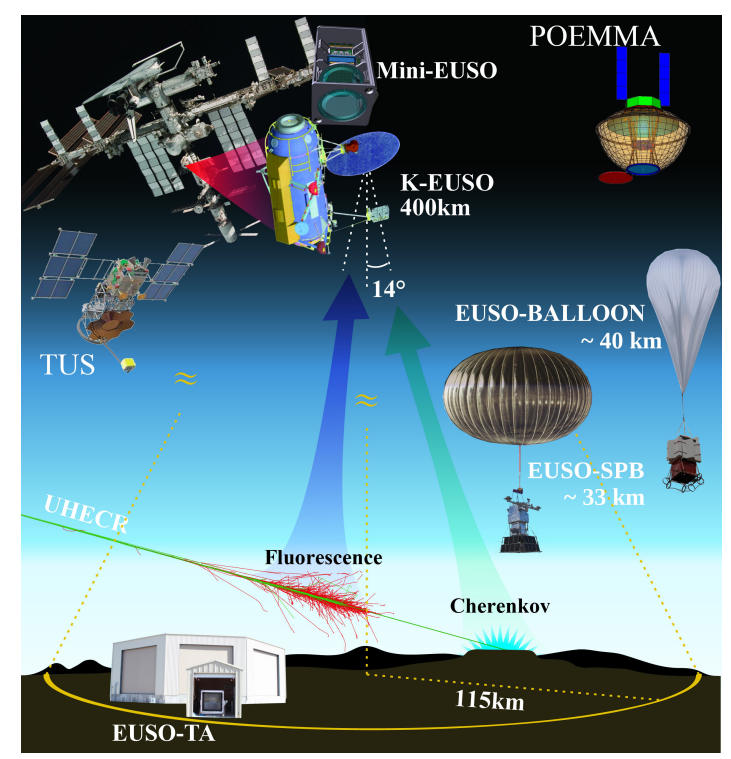

Figure 1: Roadmap of the EUSO telescopes: a) EUSO-TA: Ground detector installed in 2013 at Telescope Array site, with focal surface installed in 2015. b) EUSO-Balloon: $1^{\text {st }}$ balloon flight from Timmins, CA (French Space Agency) August 2014; c) NASA Super Pressure Balloon (SPB) flight:2017; d) TUS on Lomonosov satellite, 2016; e) MINI-EUSO (2017) inside of International Space Station (ISS); f) K-EUSO (2019): Russian section of ISS; g) POEMMA.

\section{Introduction}

The study of Ultra High Energy Cosmic Rays (UHECRs) allows to study phenomena occurring at energies currently inaccessible by man-made accelerators. Although the last years have shed light on several characteristics of these particles with $E>10^{18}$, their origin, nature and acceleration mechanisms are still unclear. A more detailed understanding of UHECR can shed light on the highest energy astrophysics and particle physics mechanisms, potentially addressing yet-unknown phenomena, such as Lorentz invariance violation. The difficulty in studying UHECRs lies in the extremely low flux of particles above $5 \times 10^{19} \mathrm{eV}$, which requires the construction of very large ground based detectors, such as Auger in the southern hemisphere and Telescope Array (TA) in the northern. However, the restriction of statistics still limits the result of these arrays, which also observe two different portion of the sky, making more difficult a comparison of the results. Spacebased UHECR observatories have the potential for a significant increase in statistics and the ability to observe the whole sky, allowing for a direct comparison of spectra and direction of arrival.

The aim of the JEM-EUSO program (Figure 1) is to bring the study of Ultra High Energy Cosmic rays (UHECR) to space. The principle of observation is based on the detection of UV light emitted by isotropic fluorescence of atmospheric Nitrogen excited by the Extensive Air Showers (EASs) in the Earth's atmosphere and forward-beamed Cherenkov radiation reflected from the Earth's surface or dense cloud tops.

A space based detector for UHECR research has the advantage of a much larger exposure and a uniform coverage of the celestial sphere. The design of a space-based telescope for UHECR 
observation has to meet strong constraints on power, mass, size and bandwidth and requires the development of a number of novel technologies, from optics to sensors, front-end and read-out electronics.

In this work we describe the current and future pathfinder missions that employ and test the principles of observation and technology of any EUSO-type mission.

\section{EUSO-TA}
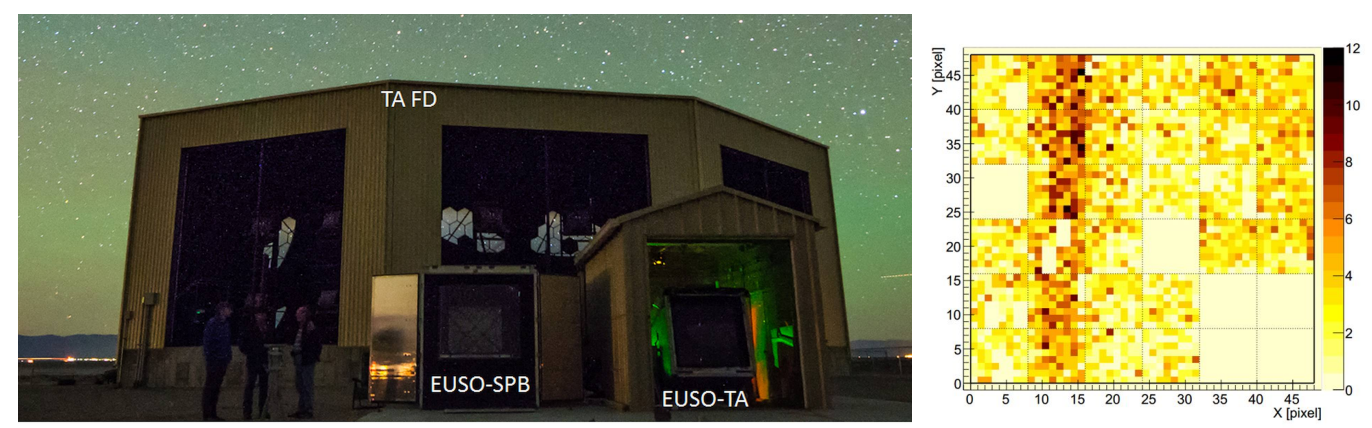

Figure 2: Left: EUSO-TA (front right), main detector of the EUSO-SPB (front left) and TAFD (back) (picture by M. Mustafa). Right: An UHECR event seen by EUSO-TA. An UHECR event of low $\left(\sim 10^{18}\right.$ $\mathrm{eV}$ ) energy traversing at $\sim 2.5 \mathrm{~km}$ distance from EUSO-TA and therefore visible as a track on a single GTU, in this case going from top-right to bottom-left (distance and energy are estimated by TA). The color scale denotes uncalibrated counts of the detector.

EUSO-TA[2] is a ground based detector located at the Telescope Array (TA) [10] site in Black Rock Mesa, Utah, USA. The aim of the project is to observe cosmic rays in conjunction with the TA fluorescence detector (TAFD). The EUSO-TA detector [20] consists of two, $1 \mathrm{~m}^{2}$ Fresnel lenses with a field of view of $11^{\circ} \times 11^{\circ}$.

The telescope is housed in a shed located in front of one of the TAFDs, pointing in the direction of the Electron Light Source (ELS) and the Central Laser Facility (CLF). The telescope can perform observations of UV light generated by cosmic ray induced EASs, stars and artificial sources such as lasers. In 2014, the telescope structure with the lenses has been used for test of the Auger-Fast detector [8]. In the 2015 campaign, observations of various stars of different magnitude and color index have been performed. Furthermore, laser and cosmic ray events - triggered by TA - have also been observed. A self-triggering algorithm employed on the 2017 balloon flight, was also tested [7].

The detector performance has been in accordance with expectations, with little (one photoelectron) electronic noise. EUSO-TA can observe stars up to $M_{B} \simeq 5.5$ based on sums of $\simeq 1000$ frames (about $3.2 \mathrm{~ms}$ observation time). While very bright stars can be seen on single frames, stacking improves the signal-to-noise ratio, with negligible star movement on the sky compared to the angular size of the pixel. The observed stars are used as point sources to study the PSF of our detector. With a fit of standard Gaussian profile we obtain PSF with average FWHM of 2.35 pixels, well within the requirements needed for UHECR showers [26]. 
So far we have observed several events with $E \sim 10^{18} \mathrm{eV}$ : due to the proximity of the event the track is going through the whole FOV in a single GTU (one frame of $2.5 \mu \mathrm{s}$ ), as shown in Figure 2 (right). The parameters were derived from TAFD which, thanks to a larger FOV and higher time-resolution, could see the shower evolution [19].

\section{The EUSO balloon flights}

The balloon flights offer a unique possibility to develop the EUSO detectors and test the observation principle in conditions similar to those encountered in space. Actually the residual atmosphere is a more demanding test of the High Voltage of the PMTs, since a discharge is more likely than in vacuum. Also the limited telemetry from the balloon (since it is necessary to assume that the payload is not recovered) requires more stringent constraints to the trigger than in case of a space based detector. In the latter case more bandwidth is generally available and - in case of the ISS - data can be physically sent to the ground on hard disks.
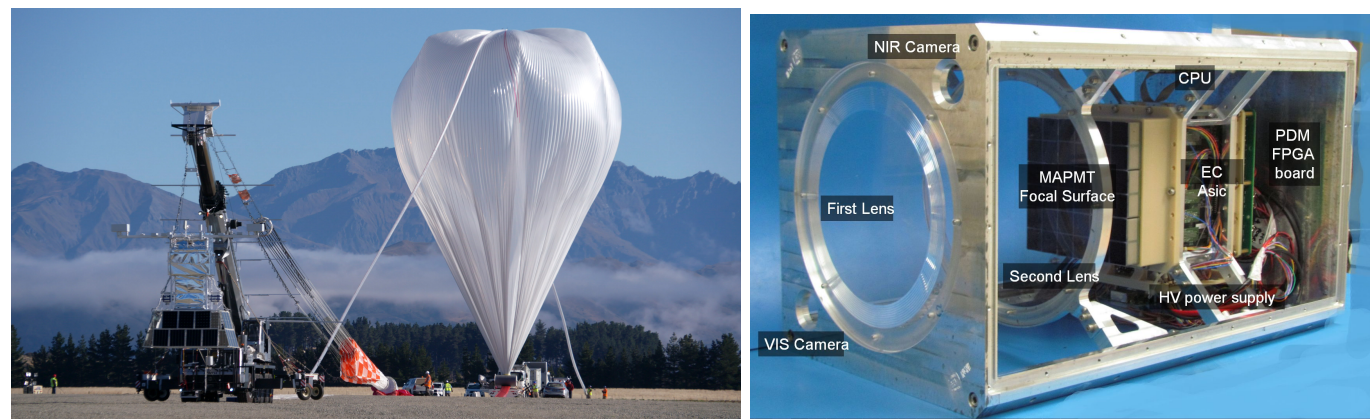

Figure 3: Left: The EUSO-SPB payload just before the flight in Wanaka, New Zealand. Right: Photo of Mini-EUSO detector. Dimensions are $35 \mathrm{~cm} \times 35 \mathrm{~cm} \times 60 \mathrm{~cm}$ (lens diameter $25 \mathrm{~cm}$ ).

The EUSO-BALLOON flight took place in August 2014 from Timmins, Canada. The CNES (French Space Agency) balloon carried an instrument similar to EUSO-TA, pointed towards nadir from a float altitude of about $40 \mathrm{~km}$. The objective of EUSO-BALLOON was to perform a full endto-end test of all the main subsystems in a near space environment. In this way it was possible to test the key technologies and methods featured in its future space mission and raise their Technological Readiness Level. The total mass of the payload was about $320 \mathrm{~kg}$. The optical bench contained two Fresnel lenses made from $8 \mathrm{~mm}$ thick PMMA with a front surface of $100 \times 100 \mathrm{~cm}$ each. The instrument booth was made as a watertight capsule using front Fresnel lens as a porthole ${ }^{1}$ [15].

EUSO-SPB was launched from New Zealand in April 2017 with a fight lasting 12 days and terminated due to a leak in the balloon. EUSO-SPB includes a new set of lenses with improved parameters and a new PDM with higher gain Photomultipliers (Figure 3). The use of a new ASIC chip - SPACIROC 3 - increases the saturation level from $\sim 30$ to more then 150 photoelectrons. The experiment houses also two auxiliary devices - an infra-red camera and a silicon photomultiplier based detector with 256 pixels. A detailed discussion of the SPB flight results can be found in [25].

\footnotetext{
${ }^{1}$ This protected the electronics in the water landing, with the payload being successfully recovered.
} 


\section{Mini-EUSO}

The Mini-EUSO space experiment ("UV atmosphere" in the Russian Space Program) is designed to perform orbital observations in UV spectrum, pointing towards Earth [21]. Mini-EUSO presents the opportunity to study a diverse range of scientific phenomena including atmospheric physics, strange quark matter, bioluminescence and search for UHECRs above $5 \times 10^{20} \mathrm{eV}$ [5]. It will also create the first night-time map of the Earth in UV light. The mission also aims to raise the technology readiness level (TRL) of any future EUSO missions to observe UHECRs from space. The Mini-EUSO measurements will be performed from the ISS through a UV transparent window in the Russian Zvezda Service Module. Furthermore, Mini-EUSO is the first step in a roadmap of potential space debris removal via laser ablation [6].

The Mini-EUSO instrument (Figure 3) comprises a compact telescope with a large field of view $\left( \pm 22^{\circ}\right.$ ), based on an optical system employing two $25 \mathrm{~cm}$ diameter Fresnel lenses (focal length $\simeq 30 \mathrm{~cm}$ ) for increased light collection [24]. The UV light is focused onto one PDM module and stored on on-board disks. The observations will be performed on $\mu$ s to seconds scales both in triggered modes (separately for fast and slow events) and "film mode" consisting of data taken in regular intervals. Launch is foreseen between Autumn 2017 and Spring 2018 in the framework of the next manned ASI (Italian Space Agency) flight, with operations planned to continue for at least three years.

\section{Moscow State University (MSU) space based experiments for UV background studies: from Tatiana to TUS}

Since 2005, Skobeltsyn Institute of Nuclear Physics of MSU made a series of experiments aimed at studying the UV radiation of the night atmosphere to estimate background and source of false triggers for UHECR detectors. This study was carried out by the UV detectors (DUV) installed on board Tatiana-1 [9], Tatiana-2 [22] and Vernov satellites [18] looking nadir.

These were followed by TUS, the first attempt to measure UHECR fluorescent light from space. TUS was launched on April 28, 2016, on a polar sun-synchronous orbit with inclination of $97.3^{\circ}$, period of $\sim 94 \mathrm{~min}$, and altitude about $500 \mathrm{~km}$. The TUS detector consists of two main parts: a parabolic mirror-concentrator of the Fresnel type and a square-shaped 256-pixel photodetector in the focal plane of the mirror. The mirror has an area of about $2 \mathrm{~m}^{2}$ with a focal distance of $1.5 \mathrm{~m}$. A pixel field of view equals $10 \mathrm{mrad}$, which results in spacial resolution of $5 \mathrm{~km}$, and the overall TUS FOV of approximately $80 \mathrm{~km} \times 80 \mathrm{~km}$ at the sea level.

During the first months of operation, the TUS detector measured numerous UV transient flashes in the EAS mode with different temporal dynamics and spatial structure. The most common type of TLEs with a specific geometry of the development in the ionosphere are so-called elves the result of the ionosphere heating by an expanding electromagnetic wave from a powerful cloudto-ground lightning. One of them was registered on September 18, 2016. A number of events are considered as EAS candidates and their detailed analyses is in progress. For more details on the TUS science results see [12]. 


\section{K-EUSO}

K-EUSO will be the first spaced-based observatory using the Earth's atmosphere as a gigantic cosmic-ray detector to perform measurements of fluxes of UHECRs. Attached to the Russian MRM-1 module on-board ISS, it will detect UHECRs above $5 \times 10^{19} \mathrm{eV}$. K-EUSO is planned to operate for a minimum of 2 years (up to 6 years, depending on the lifetime of the ISS) orbiting the Earth every 90 minutes at an altitude of about $400 \mathrm{~km}$ [13].

The K-EUSO instrument is a $4 \mathrm{~m}$ ultraviolet (UV) reflector telescope with a $14^{\circ}$ diameter field of view [23]. K-EUSO images the UV-light from the isotropic nitrogen fluorescence excited by the EASs, and the forward-beamed Cherenkov radiation reflected from the Earth's surface or dense cloud tops. A schematic of K-EUSO can be seen in Figure 6, for details on the electronics see [11]. The temporal and spatial evolution recorded by K-EUSO will allow the reconstruction of an EAS, allowing the energy and arrival direction of the UHECR to be determined. The UV images of EASs are captured by a highly-pixelized high-speed camera with a time resolution of $2.5 \mu \mathrm{s}$. The camera focal plane consists of $1.2 \times 10^{5}$ pixels, each smaller than $2.88 \times 2.88 \mathrm{~mm}$, giving a $0.066^{\circ}$ angular resolution per pixel; a pixel covers about $0.8 \mathrm{~km}$ on the surface of the Earth (for ISS altitude of 400 $\mathrm{km})$.
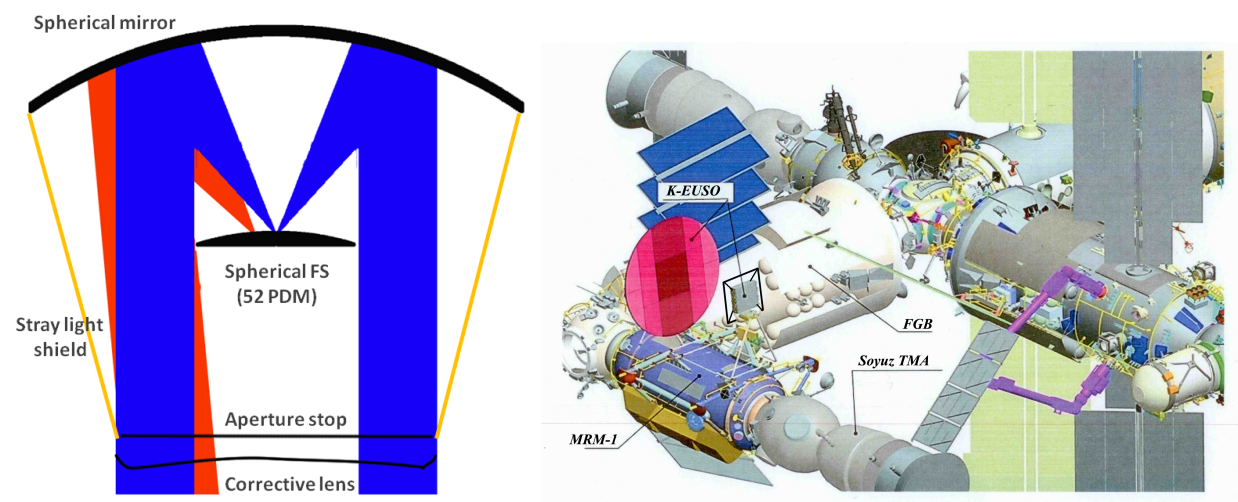

Figure 4: Left: Scheme of K-EUSO, with the spherical reflector (4m diameter) on top and corrective lens and spherical focal surface on the bottom. Right: Location of K-EUSO on the ISS.

K-EUSO will be the first detector to observe the whole UHECR sky uniformly, observing both hemispheres at the same time [4]. Furthermore, it will investigate phenomena intrinsic to the Earth's atmosphere or induced by the meteoroids coming from space. During its first year of mission, assuming TA and Auger spectra in the northern and southern hemispheres, K-EUSO will detect over 170 events above $5.7 \times 10^{19} \mathrm{eV}$ (the energy threshold used for hotspot and anisotropy search). This number is smaller than what is expected by the integrated exposure of PAO (Pierre Auger Observatory) and Telescope Array (including the future TA $\times 4$ ), but sufficient to accomplish the following two vital goals that cannot be achieved by these existing ground-based experiments alone: 1. To independently confirm (or rule out) the presence of hotspots in the northern [1] and southern hemispheres, and compare their spectral indexes. 2. To study possible UHECR anisotropies over various angular scales, including possible correlation to nearby large-scale extragalactic structures $(\approx 40$ degrees), Milky-Way related features, and dipole-type anisotropy. 


\section{POEMMA}

POEMMA is a Multi-messenger probe selected by NASA for an in-depth probe mission concept study in preparation for the next decadal survey [17]. It is a larger satellite with a foldable mirror optics. It aims to answer the following questions: What astrophysical objects can accelerate particles to ultra-high energies? How are they distributed in the sky? How strong are magnetic fields in the intergalactic medium? How does the composition of UHECRs evolve at the highest energies? What is the flux of cosmogenic neutrinos? POEMMA detector combines the Orbiting Wide-field Light collectors (OWL) concept [14], the CHerenkov from Astrophysical Neutrinos Telescope (CHANT) [16] concept and the JEM-EUSO [3] focal surface.

\section{Acknowledgments}

This work was partially supported by Basic Science Interdisciplinary Research Projects of RIKEN and JSPS KAKENHI Grant (22340063, 23340081, and 24244042), by the Italian Ministry of Foreign Affairs and International Cooperation, by the Italian Space Agency through the ASI INFN agreement $n$. 2017-8-H.0, by contract contract 2016-1-U.0, by NASA award 11-APRA0058 in the USA, by the Deutsches Zentrum für Luft- und Raumfahrt, by the French space agency CNES, the Helmholtz Alliance for Astroparticle Physics funded by the Initiative and Networking Fund of the Helmholtz Association (Germany), and by Slovak Academy of Sciences MVTS JEMEUSO as well as VEGA grant agency project 2/0132/17. Russia is supported by ROSCOSMOS and the Russian Foundation for Basic Research Grant No 16-29-13065, and the Olle Engkvist Byggmästare Foundation.

We dedicate this proceeding to Yoshiya Kawasaki and Jacek Karczmarczyk, who passed away in 2016.

\section{References}

[1] Abbasi, R. U., et al. Indications of Intermediate-scale Anisotropy of Cosmic Rays with Energy Greater Than $57 \mathrm{EeV}$ in the Northern Sky Measured with the Surface Detector of the Telescope Array Experiment. ApJL , 790:L21, August 2014. doi:10.1088/2041-8205/790/2/L21.

[2] Adams, J. H., et al. Ground-based tests of JEM-EUSO components at the Telescope Array site, "EUSO-TA". Experimental Astronomy, 40:301-314, November 2015. doi:10.1007/s10686-015-9441-6.

[3] Adams, J. H., et al. JEM-EUSO observational technique and exposure. Experimental Astronomy, 40:117-134, November 2015. doi:10.1007/s10686-014-9376-3.

[4] Casolino, M. Klypve-euso: Science and uhecr observational capabilities. These proceedings.

[5] Casolino, M. Science of mini-euso detector on board the international space station. These proceedings.

[6] Ebisuzaki, T., et al. Demonstration designs for the remediation of space debris from the international space station. Acta Astronautica, 112:102 - 113, 2015. ISSN 0094-5765.

doi:http://dx.doi.org/10.1016/j.actaastro.2015.03.004. 
[7] Fenu, F., et al. Preliminary analysis of EUSO-TA data. Journal of Physics Conference Series, 718(5):052011, May 2016. doi:10.1088/1742-6596/718/5/052011.

[8] Fujii, T., et al. Detection of ultra-high energy cosmic ray showers with a single-pixel fluorescence telescope. Astroparticle Physics, 74:64-72, February 2016. doi:10.1016/j.astropartphys.2015.10.006.

[9] Garipov, G. K., et al. Ultraviolet radiation detector of the msu research educational microsatellite Universitetskii-Tat'yana. Instruments and Experimental Techniques, 49(1):126-131, 2006. ISSN 1608-3180. doi:10.1134/S0020441206010180.

[10] Kawai, H., et al. Telescope array experiment. Nuclear Physics B - Proceedings Supplements, 175:221 - 226, 2008. ISSN 0920-5632. doi:http://dx.doi.org/10.1016/j.nuclphysbps.2007.11.002.

[11] Klimov, P. Klypve-euso photodetector design: focal surface and data processing system. These proceedings.

[12] Klimov, P. Results of the tus detector. These proceedings.

[13] Klimov, P. Status of the klypve-euso detector for uhecr study on board the iss. These proceedings.

[14] Krizmanic, J. F., et al. Optimization of the Orbiting Wide-angle Light Collectors (OWL) Mission for Charged-Particle and Neutrino Astronomy. ArXiv e-prints, July 2013.

[15] M.Bertaina. Results of the euso-balloon flight. These proceedings.

[16] Neronov, A., et al. Sensitivity of a proposed space-based Cherenkov astrophysical-neutrino telescope. Phys. Rev. D , 95(2):023004, January 2017. doi:10.1103/PhysRevD.95.023004.

[17] Olinto, A. V. Poemma: Probe of extreme multi-messenger astrophysics. These proceedings.

[18] Panasyuk, M. I., et al. RELEC mission: Relativistic electron precipitation and TLE study on-board small spacecraft. Advances in Space Research, 57:835-849, February 2016. doi:10.1016/j.asr.2015.11.033.

[19] Paul, T. Simulation study of the detected and expected events for the euso-ta fluorescence detector. These proceedings.

[20] Piotrowski, L. The euso-ta detector: status and performance. These proceedings.

[21] Ricci, M. Mini-euso, a precursor mission to observe and study atmosphere and earth uv emission from the international space station. These proceedings.

[22] Sadovnichy, V. A., et al. Investigations of the space environment aboard the Universitetsky-Tat'yana and Universitetsky-Tat'yana-2 microsatellites. Solar System Research, 45:3-29, February 2011. doi:10.1134/S0038094611010096.

[23] Sandri, P. K-euso schmidt type optical system for the klypve-euso uhecr detector. These proceedings.

[24] Takizawa, Y. Mini-euso optics design and test. These proceedings.

[25] Wiencke, L. Euso-spb mission and science. These proceedings.

[26] Z.Plebaniak. Point spread function of euso-ta detector. These proceedings. 